Research Article

\title{
De novo transcriptome assembly of the lobster cockroach Nauphoeta cinerea (Blaberidae)
}

\author{
Ana Lúcia Anversa Segatto ${ }^{1}$, José Francisco Diesel ${ }^{1}$, Elgion Lucio Silva Loreto ${ }^{1}$ and João Batista Teixeira \\ da Rocha ${ }^{1}$ \\ ${ }^{1}$ Departamento de Bioquímica e Biologia Molecular, CCNE, Universidade Federal de Santa Maria, Santa \\ Maria, RS, Brazil.
}

\begin{abstract}
The use of Drosophila as a scientific model is well established, but the use of cockroaches as experimental organisms has been increasing, mainly in toxicology research. Nauphoeta cinerea is one of the species that has been studied, and among its advantages is its easy laboratory maintenance. However, a limited amount of genetic data about $N$. cinerea is available, impeding gene identification and expression analyses, genetic manipulation, and a deeper understanding of its functional biology. Here we describe the $N$. cinerea fat body and head transcriptome, in order to provide a database of genetic sequences to better understand the metabolic role of these tissues, and describe detoxification and stress response genes. After removing low-quality sequences, we obtained 62,121 transcripts, of which more than $50 \%$ had a length of $604 \mathrm{pb}$. The assembled sequences were annotated according to their genes ontology (GO). We identified 367 genes related to stress and detoxification; among these, the more frequent were p450 genes. The results presented here are the first large-scale sequencing of $N$. cinerea and will facilitate the genetic understanding of the species' biochemistry processes in future works.
\end{abstract}

Keywords: Nauphoeta cinerea, transcriptome assembly, fat body, detoxification.

Received: August 27, 2017; Accepted: January 03, 2018.

\section{Introduction}

The understanding of biological phenomena is dependent on the organisms observed, and increasing the variety of experimental organisms can provide clearer views of the targeted processes (Golstein et al., 2003). Among the reasons that have motivated the search for alternative experimental organisms is also the pressure to reduce the use of mammalian species in toxicological testing (Peterson et al., 2008). As an alternative experimental organism, the use of the cockroach is increasing (Walz et al., 2006; Blankenburg et al., 2015). Cockroaches (order Dictyoptera) are primitive winged insects, with worldwide distribution and comprising approximately 4,000 species (Bell et al., 2007). Thirty of these species are associated with human household, and less than $1 \%$ is thought to be associated with human health problems (Fakoorziba et al., 2010).

Periplaneta americana (L.) is the most common domestic cockroach species, it shows great reproductive capability and has been used as an experimental organism in scientific studies, including neurophysiology research (Wicher et al., 2006; Nishino et al., 2010). At least three

Send correspondence to Elgion Lucio Silva Loreto. Departamento de Bioquímica e Biologia Molecular, CCNE, Universidade Federal de Santa Maria, 97105-900 Santa Maria, RS, Brazil. E-mail: elgion@base.ufsm.br. transcriptomes of different $P$. americana tissues were published (Chen et al., 2015; Zhang et al., 2016; Kim et al., 2016). Another cockroach species frequently used as scientific experimental organism is the German cockroach Blattella germanica (L.) (Cruz et al., 2006). This species is highly dependent on humans for survival, and its domestic presence is associated with the occurrence of allergic respiratory diseases (Brenner, 1995). One transcriptome of this species has been published (Zhou et al., 2014).

The lobster cockroach Nauphoeta cinerae (Olivier) has been used as an experimental model for toxicology (Rodrigues et al., 2013; Adedara et al., 2015, 2016) and shown to be a valid alternative for basic toxicological studies. Compared with the other species of cockroaches used in scientific studies, $N$. cinerea is easy to handle and does not fly, facilitating its maintenance in the laboratory. However, until now, there are few genetic sequences available for this species, and studies using molecular biology techniques like quantitative real-time PCR have been laborious to implement.

Cockroaches are found in diverse and inhospitable habitats that can have different amounts of toxic substances, such as environmental pollutants, microbial toxins, insecticides, and other xenobiotics. Thus, they may be a good experimental model to study detoxification abilities 
and stress response (Bell et al., 2007; Zhang et al., 2016). The usual response to stress conditions is the overproduction of reactive oxygen species (ROS), resulting in redox homeostasis alterations as well as oxidative stress. Overproduction of ROS have been associated to the toxicity of a wide range of xenobiotics, such as benzo[a]pyrene (Winn and Wells 1997), methamphetamine (McCallum et al., 2011; Wong et al., 2008), ethanol (Dong et al., 2008, 2010), sodium fluoride (Umarani et al., 2015; Samanta et al., 2016; Song et al., 2017), and methylmercury (Usuki and Fujimura, 2016). However, ROS are also produced by normal cellular metabolism, and one of its beneficial effects is on the organism's defense system (Valko et al., 2007).

The main components of the antioxidant system are conserved along the evolutionary process, but there are different adaptations in different groups. In insects, the major change in comparison to other phylogenetic groups is the absence of selenium-dependent glutathione peroxidase (SeGPx). It has been proposed that in insect GPxs evolution, selenium was replaced by cysteine more than once (Bae et al., 2009; Flohe et al., 2011). Due the variations among groups, the detoxification genes being expressed should be known before starting studies of exposure to toxic compounds.

Antioxidant enzymes can be divided as acting in phase I (primary) and phase II (secondary) reactions. Phase I reactions consist of oxidation, hydrolysis and reduction, and the enzymes involved are aldehyde dehydrogenases, alcohol dehydrogenases, catalases, cytochrome P450s, dehalogenases, hydroxylases, oxidoreductases, peroxidases, superoxide dismutases (SODs), thioredoxins, and glutaredoxins. Secondary antioxidant enzymes that act indirectly on ROS include acetyltransferases, acyltransferase, and glutathione S-transferases (GSTs).

The fat body, together with midgut, is the primary detoxification organ in insects, but its gene expression profile is poorly addressed. In addition, the fat body plays an essential role in most intermediary metabolism reactions, as well as energy storage and utilization (Arresse and Soulages, 2010), besides being an endocrine organ producing several antimicrobial peptides (Wen et al., 2011).

Genetic and transcriptome information of $N$. cinerae cockroaches is scarce. Transcriptome sequencing is a costeffective tool for non-model organisms and the highthroughput sequencing technologies are an efficient method for genomic characterization of a species and gene discovery (Wang et al., 2009). Here, we describe the transcriptome of $N$. cinerae fat body and head, with a focus on the detoxification and stress response genes. We also analyzed the differential gene expression of tissues to investigate the metabolic activity of the fat body and its participation in detoxification routes.

\section{Materials and Methods}

\section{Cockroach rearing, RNA isolation and Illumina sequencing}

The $N$. cinerea specimens used in this work were obtained from the Laboratório de Bioquímica Toxicologica, Universidade Federal de Santa Maria, Brazil. The insects were maintained in dark plastic boxes under standard conditions (Adedara et al., 2015). RNA was extracted from two dissected body parts: the head (all its tissues, including the central nervous system), and the fat body (containing epidermal, neuronal, muscle cells, and other integument constituents). Two and four individuals were used for head and fat body RNA extraction, respectively. Total RNA was prepared from the two tissues using Trizol Reagent (Life Technologies) according to the manufacturer's instructions. A second extraction procedure was done from the product of the first for RNA cleanup. Library preparation followed the recommendation of the TruSeq ${ }^{\circledR}$ Stranded mRNA Library Prep Kit (Illumina). Briefly, total RNA was purified and fragmented. The cleaved RNA fragments were then employed for first-strand cDNA synthesis, followed by second-strand cDNA synthesis. Fragments underwent a 3' adenylation process and were ligated with adapters before the PCR reaction was carried out. After library validation, the products were normalized and pooled. The MiSeq Reagent Kit v3 (150 cycles) was employed for sequence in a Illumina MiSeq system, resulting in pair-end reads of 75 pb. We used $25 \%$ of the flow cell for each sample. Sequencing was carried out using an Illumina MiSeq Plataform by Unidade de Genôminca Computacional Darcy Fontoura de Almeida/LNCC/Brazil.

\section{Read processing, de novo transcriptome assembly, and annotation}

Read quality was checked and visualized using FastQC v 0.11.4 (Andrews, 2010), and low quality reads and adapters were eliminated using Trim Galore v 0.4.1 (Wu et al., 2011). Reads with a quality threshold of less than 25 on the Phred scale, empty reads, and short sequences with a length of less than $25 \mathrm{nt}$ were removed. The de novo assembly was performed with Trinity v 2.2.0 software using default settings (Grabherr et al., 2011). The Deconseq Standalone v 0.4.3 program (Schmieder and Edwards, 2011) was used to remove contamination from virus, bacteria, and human sequences, and contigs with such hits were removed from the assembly before further analysis. We performed three assemblies: one from head reads, one from fat body reads, and one combining all the reads. The latter was used as a reference in the transcript quantification and differential expression analysis. Functional annotation was performed using Trinotate v 3.0.1 (Haas et al., 2013 ) with a cutoff E-value of $10^{-5}$. CateGOrizer v $3.21(\mathrm{Hu}$ et al., 2008) was used to map GO terms to GO Slim file by single count in order to get a broad overview of the func- 
tional classification of the transcripts. Each GO term was manually assigned to a consensus functional class. The assembly quality was assessed by examining the percentage of input RNA-Seq reads that are represented in the transcriptome assembly. The representation of full-length reconstructed protein-coding genes was evaluated by searching the assembled transcripts against SwissProt (E-value $10^{-20}$ ) and calculating the values of $\mathrm{Nx}$ statistics. Also, in order to verify the quality of our transcriptome and the similarity among transcripts of different species of cockroaches, we used our reference transcriptome as query and performed a blastn search using $P$. americana (Chen $e t$ al., 2015) or B. germanica (Zhou et al., 2014) transcriptomes as target database. To this end, we downloaded the raw reads of $P$. americana and $B$. germanica and used Trinity $\mathrm{v}$ 2.2.0 (Grabherr et al., 2011) for the assembly, just as done for our data. The online tool BioVenn (Hulsen et al., 2008) was used to draw a Venn diagram showing the distribution of transcripts detected in the head and fat body by a blastx, with a cutoff E-value of $10^{-5}$, using the UniProt database as subject (The UniProt Consortium, 2017).

\section{Transcript quantification and differential expression analysis}

The assembly with reads from the head and fat body was used as a reference to estimate transcript abundance and build a counts matrix for each of the transcripts in each tissue using RSEM v 1.2.27 software (Li and Dewey, 2011). The expression abundance of isoforms was normalized using the TPM (transcripts per million) method (Wagner et al., 2012). Differential expression analyses were carried out using the dgeR Bioconductor package (Robinson et al., 2010) using a false discovery rate (FDR) cutoff of 0.001. Isoforms up-regulated in each sample were isolated and analyzed using Perl scripts and annotation was carried out using Blast2GO (Conesa et al., 2005) using a cutoff E-value of $10^{-5}$. CateGOrizer v 3.21 (Hu et al., 2008) was used to map GO terms to GO Slim file by single count.

\section{Identification and analysis of genes of interest}

To directly identify detoxification and stress response genes in our transcriptome, we translated our transcripts in the six frames using the EMBOSS Transeq command line (Rice et al., 2000; Goujon et al., 2010), and used this as a database in blast + using blastx (Camacho et al., 2008). We searched for genes related to oxidation and reduction, conjugation, hydrolysis, and other functions possibly related to stress (Xu et al., 2013) in Flybase to then be used as a query.

\section{Results}

\section{De novo assembly of an $N$. cinerea transcriptome}

A total of 24,980,364 Illumina MiSeq reads from fat body and head tissue were generated. The SRA submission reads can be found under the accession numbers
SRR3581673 and SRR3581312. After trimming, $23,137,682$ reads were assembled in a reference transcriptome, combining head and fat body reads, this resulting in 62,121 transcripts with an N50 of 604 (Table 1). The separated assemblies of the head and fat body transcriptomes (Table 1) were used in transcript quantification and the differential expression analysis. To assess the quality of our assembly we also mapped the reads back to the transcripts and found that among $70-80 \%$ of the fragments were mapped as proper pairs. In each assembly, approximately 2,000 transcripts are represented by nearly full-length proteins, having $>80 \%$ alignment coverage.

\section{Functional annotation of the reference transcriptome}

The transcriptome was annotated using Trinotate v3.0.1 (Haas et al., 2013). Blast homologies were captured by searching the UniProt/Swiss-Prot protein databases for further GO assignments. Mapping Entrez Gene IDs to GO annotations identified 44,963 terms categorized in 114 functional groups. The GO terms attributed to the greatest number of genes, in descending order, were metabolism (GO:0008152), catalytic activity (GO:0003824), and development (GO:0007275) (Figure 1). Twelve GOs were classified with antioxidant activity and 558 as response to stress activity. The assembly of $P$. americana resulted in 158,464 transcripts with a N50 of 536 and the assembly of $B$. germanica resulted in 71,903 transcripts with a N50 of 904. Our assembly strategy resulted in more transcripts and a higher N50 for B. germanica than previously described (Zhou et al., 2014). For P. americana, our assembly strategy resulted in more transcripts but with a smaller average size than that previously described (Chen et al., 2015). A total of $533 N$. cinerea transcripts showed a Blast similarity with $P$. americana, and 2,679 with B. germanica with an E-value of zero. The top 10 hits of blastn results, with lower E-value and higher bit score are listed in Supplementary Information Table S1. The percentage of GC was similar in all the cockroach transcriptomes assembled, being, near 37.94 in $N$. cinerea fat body and 40.08 in $P$. americana testis.

\section{Differential expression}

Using a dispersion of 0.1 in edgeR (Robinson et al., 2010 ) we found 3,257 isoforms as differentially expressed

Table 1 - Summary statistics of transcriptome assembly.

\begin{tabular}{lccc}
\hline & Head & Fat body & Reference \\
\hline Number of reads & $12,935,304$ & $12,045,060$ & $24,980,364$ \\
Reads after trimming & $6,098,525$ & $5,470,316$ & $23,137,682$ \\
Total Trinity genes & 41,631 & 27,568 & 57,928 \\
Total Trinity transcripts & 43,991 & 29,163 & 62,121 \\
Percent CG & 38.78 & 37.94 & 38.38 \\
Total assembled bases & $21,525,406$ & $13,939,303$ & $31,375,798$ \\
Transcript contig N50 & 576 & 547 & 604 \\
\hline
\end{tabular}


in the head and fat body of the lobster cockroach. The up-regulated isoforms in the head were classified in 1,749
GO terms and could be categorized into 77 functional groups. In the fat body, a total of $864 \mathrm{GO}$ terms were cate-
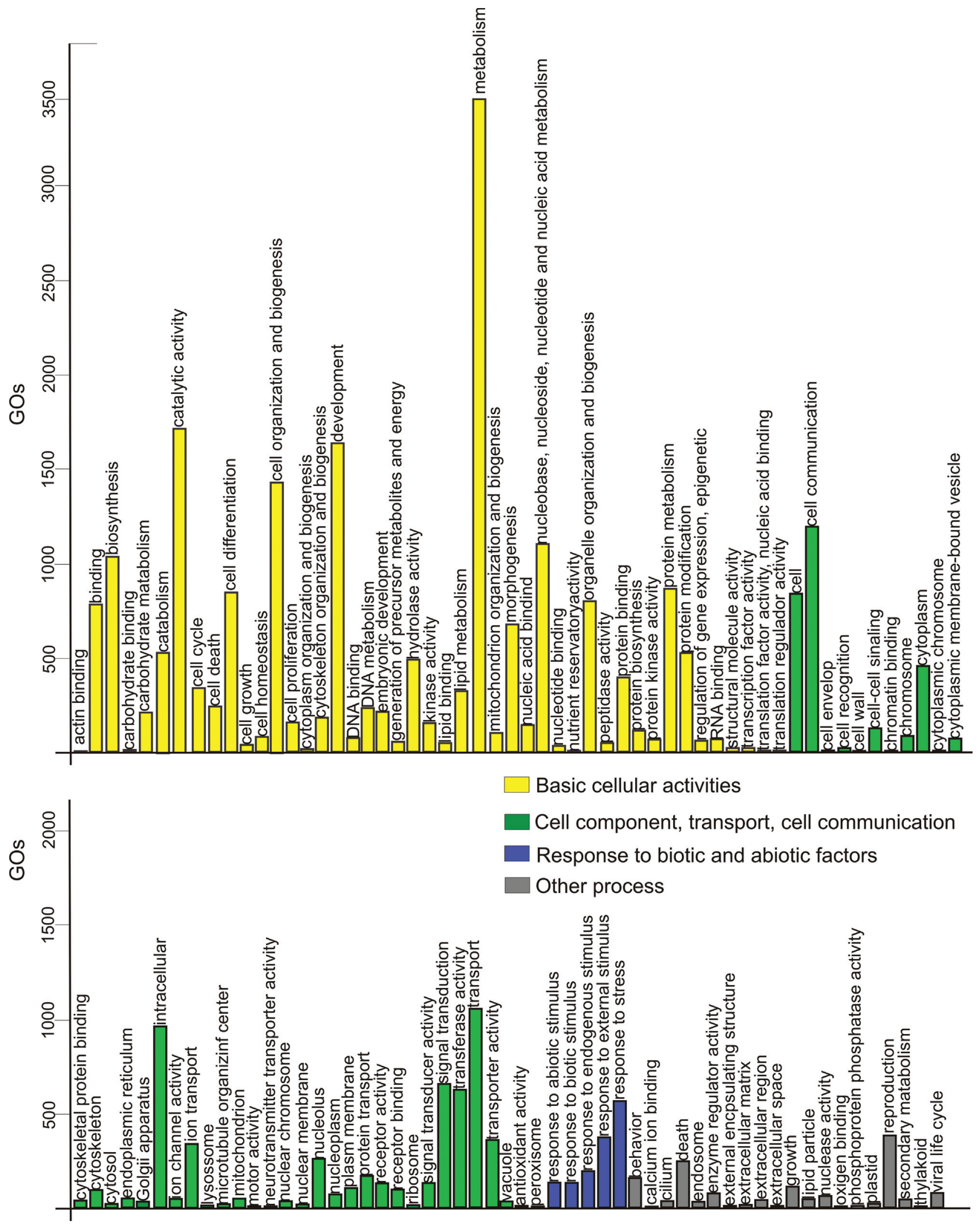

Figure 1 - GO slim terms distribution associated with the $N$. cinerea transcriptomes. 

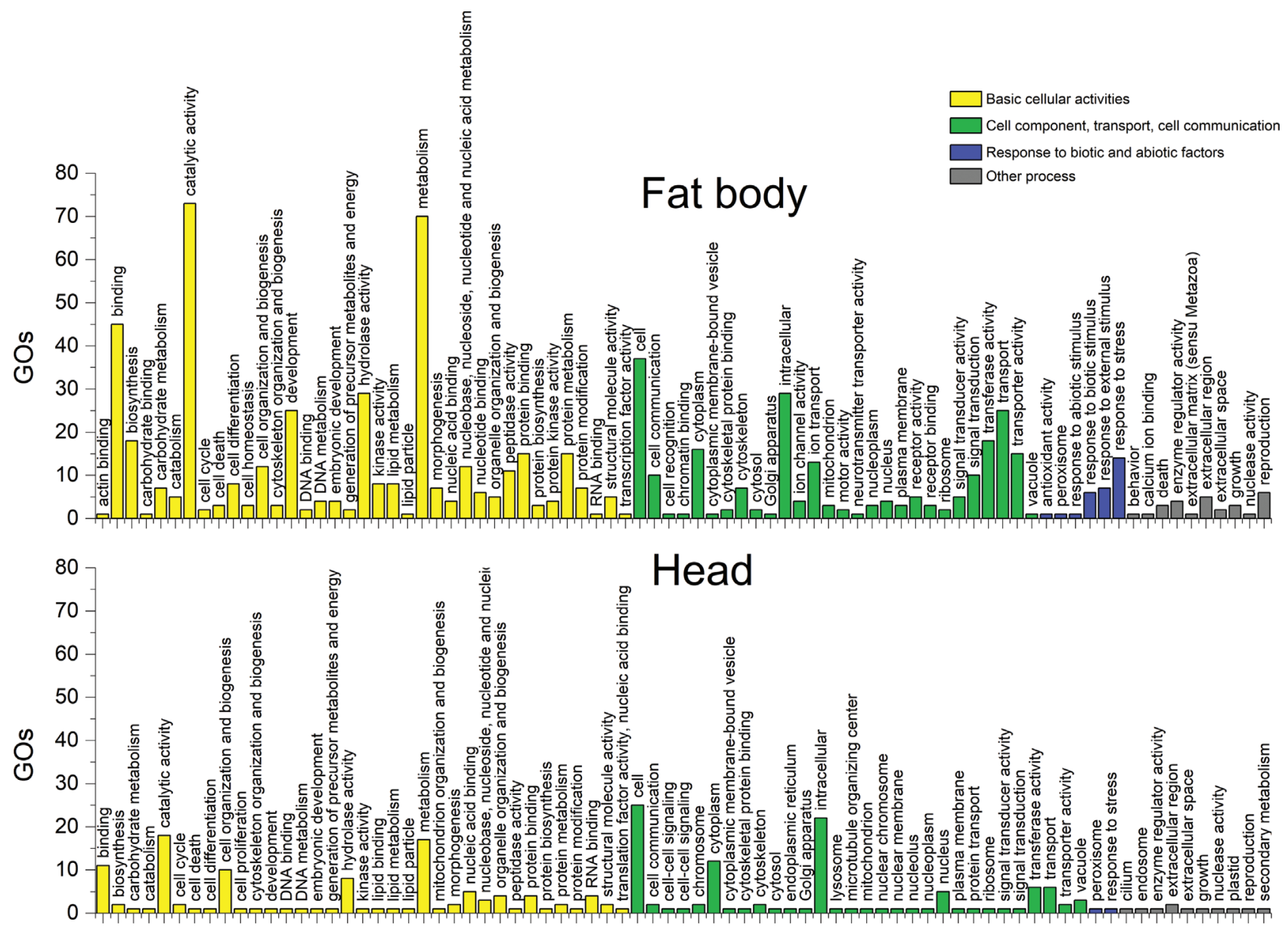

Figure 2 - GO slim terms distribution of upregulated genes in the fat body and head of $N$. cinerea.

gorized into 81functional groups (Figure 2). Regarding species distribution, the top hit species was Zootermopsis nevadensis Hagenfor. Blattella germanica, and $P$. americana appeared among the six top hits in both tissues. Among the classification of up-regulated genes in the fat body, 30 GOs were associated with response to biotic and abiotic factors, while only 10 up-regulated genes were found in the head in this subcategory. Differentially expressed transcripts are shown in red in the volcano plot of Figure 3A. From the total of 43,991 transcripts assembled in the head and 29,163 assembled in the fat body, 37,743 and 24,174 had blastx results respectively, using the UniProt database as subject and an E-value of $10^{-5}$ as cut-off. After removing redundant hits, 5,921 Blast results were common between the assembled transcriptomes (Figure 3B).

\section{Detoxification and stress genes}

We identified 367 transcripts that were classified into four main categories: 1) oxidation and reduction, 2) conjugation, 3) hydrolytic enzymes, and 4) other transcripts with function possibly related to stress. In each category, the most abundant subcategory is presented in Table 2. Among the oxidation and reduction enzymes, cytochrome p450 genes were the most abundant ones. Glycosyl transferases were the most abundant transcripts among the conjugation enzymes. Acetylcholine and carboxyl esterases were the most frequently found hydrolytic enzyme transcripts. Heat shock proteins were also found in substantial numbers.

\section{Discussion}

After cleaning and quality checks, we obtained 1.7 $\mathrm{Gb}$ of reads. Nauphoeta cinerea has a C value (pg) of 5.15, or a genome size of approximately $5,000 \mathrm{Mb}$ (Koshikawa et $a l$., 2008). Hence, our transcriptome, disregarding isoforms, represented around $30 \%$ of the $N$. cinerea genome. Our library was enriched for mRNA sequences, and the reads obtained were $75 \mathrm{bp}$ long, sequenced as pair-end. Thus, it was necessary to reconstruct full-length transcripts by transcriptome assembly. A transcriptome assembly encounters many challenges, among them differential expression of transcripts and alternative splicing (Grabherr et al., 2011). In spite of these challenges, the comparison with other cockroaches transcriptomes (Zhou et al., 2014, Chen et al., 2015; Kim et al., 2016) showed that we had obtained a good assembly with a relatively small amount of data us- 


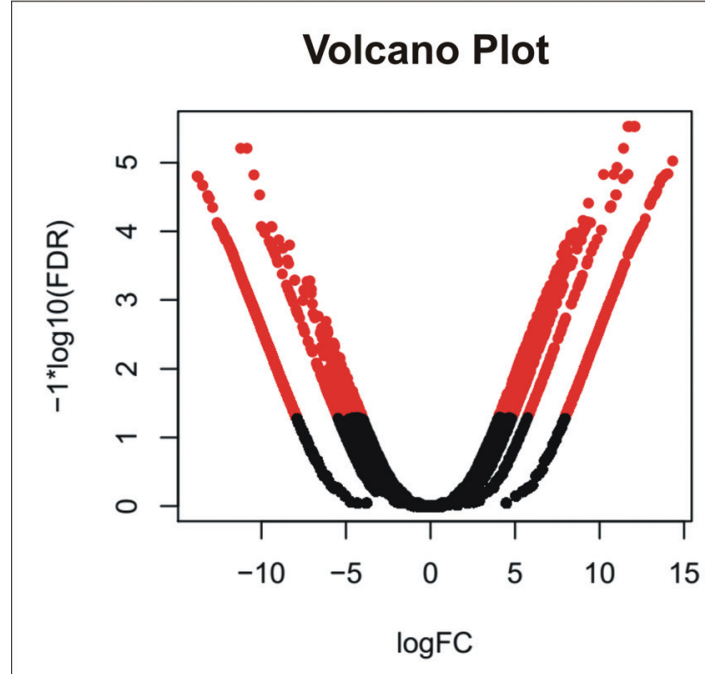

\section{Venn diagranm}

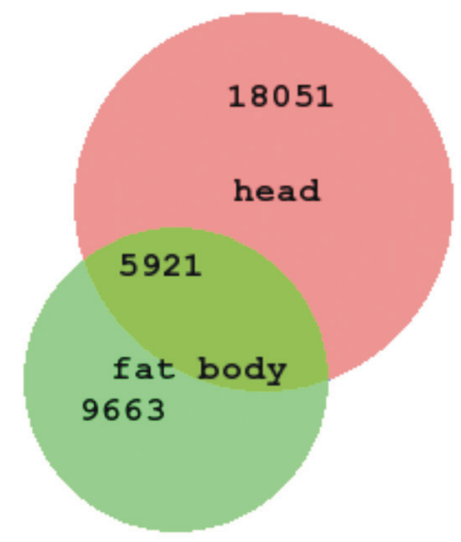

A

B

Figure 3 - Comparison among head and fat body transcripts. (A) Volcano plot of differentially expressed transcripts; the X-axis displays the fold change expression differences (FC) and the Y-axis the statistical significance based on a false discovery rate (FDR) cut-off of 0.001. (B) Venn diagram of blastx results of transcripts detected in the head and fat body.

Table 2 - Detoxification genes expressed in the reference transcriptome.

\begin{tabular}{lc}
\hline Detoxification gene transcripts & Numbers found \\
\hline $\begin{array}{lc}\text { Oxidation and reduction en- } \\
\text { zymes }\end{array}$ & $\mathbf{1 3 2}$ \\
Cytochrome P450s & 85 \\
Conjugation enzymes & $\mathbf{1 0 3}$ \\
Glycosyl transferases & 37 \\
Hydrolytic enzymes & $\mathbf{6 9}$ \\
Acetylcholine and carboxyl ester- & 28 \\
ases & \\
Possible stress related functions & $\mathbf{6 3}$ \\
Heatshock proteins & 29 \\
\hline
\end{tabular}

ing Trinity v2.2.0 (Table 1). An outstanding characteristic of the cockroach transcriptomes that we assembled was the low level of GC content. A high GC content is correlated to high recombination rate, and in insects genomes, the GC content is usually low, but can be heterogeneous (Kent et al., 2012; Kent and Zayed, 2013).

Blastn was used to find similarities between $N$. cinerea and the other cockroach transcriptomes, and large structural proteins gave among the best results (Table S1). The $B$. germanica assembly has overall better Blast results, which may be a consequence of its higher N50 provided by that the Roche 454 sequencing method, which produces longer reads that can improve the assembly in complex regions (Martin and Wang, 2011). Here, we used as a strategy a small number of individuals for RNA extraction, which on the one hand, simplifies the assembly as a result of less genetic variations, but on the other rules out the possibility of performing any population analysis or searches for SSR markers and SNPs.
The transcriptome annotation showed that the most frequent GOs (Figure 1 and 2) are similar to other insect transcriptomes (Zhou et al., 2014, Chen et al., 2015; Wadsworth and Dopman 2015; Kim et al., 2016; Zhang et al., 2016). The annotation also revealed a high similarity of the $N$. cinerea sequences with the termite Zootermopsis nevadensis (Blattodea). While this can be related to the amount of sequences available in databases, the phylogenetic relationship between termites and cockroaches is still controversial (Legendre et al., 2015).

Up-regulated genes were more frequent in fat body, confirming the versatility of this organ in insects (Arresse and Soulages, 2010), even when compared to a tissue set that contains sensory organs and central nervous system ganglia (Figure 2). In addition, there were only 5,921 common Blast results among these tissues, in a total of 39,553 Blast results (Figure 3B). These numbers reflect the big functional difference among head and fat body tissue. It is important to note that although the fat body had a lesser number of assembled transcripts, it had more up-regulated genes in comparison with the head tissue.

The individuals used to generate the transcriptomes had not been submitted to any specific stress condition. Consequently, the elevated number of genes related to biotic and abiotic stress in the differential expression analysis confirm the role of the fat body as an active detoxification organ (Figure 2). Our interest in detoxification genes is due to the growing use of $N$. cinerea as a potential model for toxicological biochemistry studies. In a study aiming to identify candidate genes for insecticide resistance in insecticide susceptible and resistant strains of Anopheles gambiae, no single body part (including the fat body) emerged as the key site of overtranscription of putative insecticide resistance genes (Ingham et al., 2014). In contrast, 
our result indicate a quite different pattern, with genes up-regulated in the fat body compared to head tissue in specimens maintained in the laboratory. It is important to highlight that the heads and fat bodies used for RNA extraction were from different individuals. A more comprehensive study design involving multiple dissected tissues and individuals exposed to different stress conditions would facilitate the comprehension of the role of the fat body in biotic and abiotic stress responses. It is well known that the fat body and hemocytes are the major components of the innate immune response in insects. Signals resulting from such stimuli can activate the synthesis and secretion of antimicrobial peptides by the fat body (Tsakas and Marmaras, 2010). However, the metabolic response of the fat body to ROS and the activation of inflammation-associated signaling pathways remains to be determined (Gloire et al., 2006).

In the reference assembly, we found many genes related to detoxification, in similar number to those found in transcriptomes of other insects (Xu, et al., 2013), indicating that our assembly strategy was efficient. Consequently, the sequences obtained here are a valuable source for future studies of such genes in $N$. cinerea. Detoxification related cytochrome p450 transcripts were found in the highest number (85). The termite Zootermopsis nevadensis genome has 76 p450 genes (Terrapon et al., 2014). In contrast, a search for detoxification and insecticide target genes in B. germanica, resulted in $163 \mathrm{p} 450$-related genes (Zhou et al., 2014). A similar search previously done on the midgut transcriptome of $P$. americana resulted in $31 \mathrm{P} 450$ transcripts (Zhang et al., 2016). It is important to note that these results were obtained in transcriptome data that can both subestimate and overestimate this diversity compared to genomic analyses.

In conclusion, we obtained a total of 24,980,364 reads and 57,928 genes, constituting a public database for gene identification and expression analysis in $N$. cinerea. The data presented here are a starting point to understand the fat body metabolism of $N$. cinerea based on nucleic acid sequences. In addition, our results attest to the multifunctionality of the fat body in insects.

\section{Acknowledgments}

This project was financially supported by the Conselho Nacional de Desenvolvimento Científico e Tecnológico $(\mathrm{CNPq})$, the Coordenação de Aperfeiçoamento de Pessoal de Nível Superior (CAPES), and the Fundação de Amparo à Pesquisa do Estado do Rio Grande do Sul (FAPERGS/Pronex 16/2551 - 0000499-4).

\section{References}

Adedara IA, Rosemberg DB, Souza DO, Kamdem JP, Farombi EO, Aschner M and Rocha JB (2015) Biochemical and behavioral deficits in the lobster cockroach Nauphoeta cinerea model of methylmercury exposure. Toxicol Res 4:442-451.
Adedara IA, Rosemberg DB, Souza DO, Kamdem JP, Farombi EO, Aschner M and Rocha JB (2016) Neuroprotection of luteolin against methylmercury-induced toxicity in lobster cockroach Nauphoeta cinerea. Environ Toxicol Pharmacol 42: 243-251.

Arresse EL and Soulages JL (2010) Insect fat body: Energy, metabolism, and regulation. Annu Rev Entomol 55:207-225.

Bae YA, Cai GB, Kim SH, Zo YG and Kong Y (2009) Modular evolution of glutathione peroxidase genes in association with different biochemical properties of their encoded proteins in invertebrate animals. BMC Evol Biol 9:72.

Bell WJ, Roth LM and Nalepa CA (2007) Cockroaches: ecology, behavior, and natural history. The Johns Hopkins University Press, Baltimore, $248 \mathrm{p}$.

Blankenburg S, Balfanz S, Hayashi Y, Shigenobu S, Miura T, Baumann O, Baumann A and Blenau W (2015) Cockroach GABAB receptor subtypes: molecular characterization, pharmacological properties and tissue distribution. Neuropharmacology 88:134-144.

Brenner RJ (1995) Economics and medical importance of German cockroaches. In: Rust MK, Owens JM and Reierson DA (eds) Understanding and Controlling the German Cockroach. Oxford Press, New York, pp 77-92.

Camacho C, Coulouris G, Avagyan V, Ma N, Papadopoulos J, Bealer K and Madden TL (2008) BLAST+: Architecture and applications. BMC Bioinformatics 10:421.

Chen W, Liu Y and Jiang G (2015) De novo assembly and characterization of the testis transcriptome and development of EST-SSR markers in the cockroach Periplaneta americana. Sci Rep 5:11144.

Conesa A, Götz S, García-Gómez JM, Terol J, Talón M and Robles M (2005) Blast2GO: A universal tool for annotation, visualization and analysis in functional genomics research. Bioinformatics 21:3674-3676.

Cruz J, Mané-Padrós D, Bellés X and Martín D (2006) Functions of the ecdysone receptor isoform-A in the hemimetabolous insect Blattella germanica revealed by systemic RNAi in vivo. Dev Biol 297:158-171.

Dong J, Sulik KK and Chen SY (2008) Nrf2-mediated transcriptional induction of antioxidant response in mouse embryos exposed to ethanol in vivo: Implications for the prevention of fetal alcohol spectrum disorders. Antioxid Redox Signal 10:2023-2033.

Dong J, Sulik KK and Chen SY (2010) The role of NOX enzymes in ethanol-induced oxidative stress and apoptosis in mouse embryos. Toxicol Lett 193:94-100.

Fakoorziba RM, Eghbal F, Hassanzadeh J and Moemenbellahfard MD (2010) Cockroaches (Periplaneta americana and Blattella germanica) as potential vectors of the pathogenic bacteria found in nosocomial infections. Ann Trop Med Parasitol 104:521-528.

Flohe L, Toppo S, Cozza G and Ursini F (2011) A comparison of thiol peroxidase mechanisms. Antioxid. Redox Signal 15:763e780.

Gloire G, Legrand-Poels S and Piette J (2006) NF- $\kappa$ B activation by reactive oxygen species: Fifteen years later. Biochem Pharmacol 7:1493-1505.

Golstein P, Aubry L and Levraud J (2003) Cell-death alternative model organisms: Why and which? Nat Rev 4:1-10.

Goujon M, Goujon M, McWilliam H, Li W, Valentin F, Squizzato S, Paern J and Lopez R (2010) A new bioinformatics analy- 
sis tools framework at EMBL-EBI. Nucleic Acids Res 38:W695-W699.

Grabherr MG, Haas BJ, Yassour M, Levin JZ, Thompson DA, Amit I, Adiconis X, Fan L, Raychowdhury R, Zeng Q et al. (2011) Full-length transcriptome assembly from RNA-Seq data without a reference genome. Nat Biotechnol 29:644-652.

Haas BJ, Papanicolaou A, Yassour M, Grabherr M, Blood PD, Bowden J, Couger MB, Eccles D, Li B, Lieber M et al. (2013) De novo transcript sequence reconstruction from RNA-seq using the Trinity platform for reference generation and analysis. Nature Protocols 8:1494-1512.

Hu Z, Bao J and Reecy JM (2008) CateGOrizer: A web-based program to batch analyze gene ontology classification categories. Online J Bioinf 9:108-112.

Hulsen T, Vlieg J and Alkema W (2008) BioVenn - a web application for the comparison and visualization of biological lists using area-proportional Venn diagrams. BMC Genomics 9:488.

Ingham VA, Jones CM, Pignatelli P, Balabanidou V, Vontas J, Wagstaff SC, Moore GD and Ranson H (2014) Dissecting the organ specificity of insecticide resistance candidate genes in Anopheles gambiae: Known and novel candidate genes. BMC Genomics 15:1018.

Kent FC and Zayed A (2013) Evolution of recombination and genome structure in eusocial insects. Commun Integr Biol 6:e22919.

Kent FC, Minaei S, Harpur BA and Zayed A (2012) Recombination is associated with the evolution of genome structure and worker behavior in honey bees. Proc Natl Acad Sci U S A 30:18012-18017.

Kim IW, Lee JH, Subramaniyam S, Yun E, Kim I, Park J and Hwang JS (2016) De novo transcriptome analysis and detection of antimicrobial peptides of the American cockroach Periplaneta americana (Linnaeus). PLoS One 11:e0155304.

Koshikawa S, Miyazaki S, Cornette R, Matsumoto T and Miura T (2008) Genome size of termites (Insecta, Dictyoptera, Isoptera) and wood roaches (Insecta, Dictyoptera, Cryptocercidae). Naturwissenschaften 95:859-867.

Legendre F, Nel A, Svenson G, Robillard T, Pellens R and Grandcolas P (2015) Phylogeny of Dictyoptera: Dating the origin of cockroaches, praying mantises and termites with molecular data and controlled fossil evidence. PLoS One 10:e130127.

Li B and Dewey CN (2011) RSEM: Accurate transcript quantification from RNASeq data with or without a reference genome. BMC Bioinformatics 12:323

Martin JA and Wang Z (2011) Next-generation transcriptome assembly. Nat Rev 12:671-682.

McCallum GP, Wong AW and Wells PG (2011) Cockayne syndrome $\mathrm{B}$ protects against methamphetamine-enhanced oxidative DNA damage in murine fetal brain and postnatal neurodevelopmental deficits. Antioxid Redox Signal 14:747-756.

Nishino H, Yoritsune A and Mizunami M (2010) Postembryonic development of sexually dimorphic glomeruli and related interneurons in the cockroach Periplaneta americana. Neurosci Lett 469:60-64.

Peterson RT, Nass R, Boyd WA, Freedman JH, Dong K and Narahashi T (2008) Use of non-mammalian alternative models for neurotoxicological study. NeuroToxicology 29:546-555.

Rice P, Longden I and Bleasby A (2000) EMBOSS: The European Molecular Biology Open Software Suite. Trends Genet 16:276-277.

Robinson MD, McCarthy DJ and Smyth GK (2010) edgeR: A Bioconductor package for differential expression analysis of digital gene expression data. Bioinformatics 26:139-140.

Rodrigues NR, Nunes MEM, Silva DGC, Zemolin APP, Meinerz DF, Cruz LC, Perreira AB, Rocha JB, Posser T and Franco JL (2013) Is the lobster cockroach Nauphoeta cinerea a valuable model for evaluating mercury induced oxidative stress? Chemosphere 92:1177-1182.

Samanta A, Chanda S, Bandyopadhyay B and Das N (2016) Establishment of drug delivery system nanocapsulated with an antioxidant (1)-catechin hydrate and sodium meta borate chelator against sodium fluoride induced oxidative stress in rats. J Trace Elem Med Biol 33:54-67.

Schmieder R and Edwards R (2011) Fast identification and removal of sequence contamination from genomic and metagenomic datasets. PLoS One 6:e17288.

Song C, Zhao J, Fu B, Li D, Mao T, Peng W, Wu H and Zhang Y (2017) Melatonin-mediated upregulation of Sirt3 attenuates sodium fluoride-induced hepatotoxicity by activating the MT1-PI3K/AKT-PGC-1 $\alpha$ signaling pathway. Free Radic Biol Med 112:616-630.

Terrapon N, Li C, Robertson HM, Ji L, Meng X, Booth W, Chen Z, Childers CP, Glastad KM, Gokhale K et al. (2014) Molecular traces of alternative social organization in a termite genome. Nat Commun 5:3636.

The UniProt Consortium (2017) UniProt: The universal protein knowledgebase. Nucleic Acids Res 45:D158-D169.

Tsakas S and Marmaras VJ (2010) Insect immunity and its signalling: An overview. Invertebr Survival J 7:228-238.

Umarani V, Muvvala S, Ramesh A, Lakshmi BV and Sravanthi N (2015) Rutin potentially attenuates fluoride-induced oxidative stress-mediated cardiotoxicity, blood toxicity and dyslipidemia in rats. Toxicol Mech Methods 25:143-149.

Usuki F and Fujimura M (2016) Decreased plasma thiol antioxidant barrier and selenoproteins as potential biomarkers for ongoing methylmercury intoxication and an individual protective capacity. Arch Toxicol 90:917-926.

Valko M, Leibfritz D, Moncol J, Cronin MTD, Mazur M and Telser J (2007) Free radicals and antioxidants in normal physiological functions and human disease. Int J Biochem Cell Biol 39:44-84.

Wadsworth CB and Dopman EB (2015) Transcriptome profiling reveals mechanisms for the evolution of insect seasonality. $\mathrm{J}$ Exp Biol 218:3611-3622.

Wagner GP, Kin K and Lynch VJ (2012) Measurement of mRNA abundance using RNA-seq data: RPKM measure is inconsistent among samples. Theory Biosci 131:281-285.

Walz B, Baumann O, Krach C, Baumann A and Blenau W (2006) The aminergic control of cockroach salivary glands. Arch Insect Biochem Physiol 62:141-152.

Wang Z, Gerstein M and Snyder M (2009) RNA-Seq: A revolutionary tool for transcriptomics. Nat Rev Genet 10:57-63.

Wen Z, Zhang X and Zhang Y (2011) P450-mediated insecticide detoxification and its implication in insecticide efficacy. In: Liu T and Kang L (eds) Recent Advances in Entomological 
Research From Molecular Biology to Pest Management. Springer, Heidelberg, pp. 229-245.

Wicher D, Agricola H, Söhler S, Gundel M, Heinemann SH, Wollweber L, Stengl M and Derst C (2006) Differential receptor activation by cockroach adipokinetic hormones produces differential effects on ion currents, neuronal activity, and locomotion. J Neurophysiol 95:2314-2325.

Winn LM and Wells PG (1997) Evidence for embryonic prostaglandin $\mathrm{H}$ synthase-catalyzed bioactivation and reactive oxygen speciesmediated oxidation of cellular macromolecules in phenytoin and benzo[a]pyrene teratogenesis. Free Radic Biol Med 22:607-621.

Wong AW, McCallum GP, Jeng W and Wells PG (2008) Oxoguanine glycosylase 1 protects against methamphetamineenhanced fetal brain oxidative DNA damage and neurodevelopmental deficits. J Neurosci 28:9047-9054.

Wu Z, Wang X and Zhang X (2011) Using non-uniform read distribution models to improve isoform expression inference in RNA-seq. Bioinformatics 27:502-508.

Xu J, Strange JP, Welker DL and James RR (2013) Detoxification and stress response gene expressed in a western North American bumble bee, Bombus huntii (Hymenoptera: Apidae). BMC Genomics 14:874.
Zhang J, Zhang Y, Li J, Liu M and Liu Z (2016) Midgut transcriptome of the cockroach Periplaneta americana and its microbiota: Digestion, detoxification and oxidative stress response. PLoS One 6:e0155254.

Zhou X, Qian K, Tong Y, Zhu JJ, Qiu X and Zeng X (2014) De novo transcriptome of the hemimetabolous German cockroach (Blattella germanica). PLoS One 9:e106932.

\section{Internet Resources}

Andrews S (2010) FastQC: A quality control tool for high throughput sequence data. http://www.bioinformatics.babraham.ac.uk/projects/fastqc.

\section{Supplementary material}

The following online material is available for this article: Table S1 - Blastn results among cockroaches transcriptomes.

Associate Editor: Houtan Noushmehr

License information: This is an open-access article distributed under the terms of the Creative Commons Attribution License (type CC-BY), which permits unrestricted use, distribution and reproduction in any medium, provided the original article is properly cited. 